\title{
Mate similarity in foraging Kerguelen shags: a combined bio-logging and stable isotope investigation
}

\author{
Elodie C. M. Camprasse ${ }^{1, *}$, Yves Cherel ${ }^{2}$, John P. Y. Arnould ${ }^{1}$, Andrew J. Hoskins ${ }^{3}$, \\ Paco Bustamante ${ }^{4}$, Charles-André Bost ${ }^{2}$
}

\begin{abstract}
${ }^{1}$ School of Life and Environmental Sciences (Burwood Campus), Deakin University, Geelong, 3220 Victoria, Australia ${ }^{2}$ Centre d'Etudes Biologique de Chizé (CEBC), UMR 7372 CNRS-Université de La Rochelle, 79360 Villiers-en-Bois, France ${ }^{3}$ CSIRO Land and Water, Canberra, 2601 Australian Capital Territory, Australia

${ }^{4}$ Littoral Environnement et Sociétés (LIENSs), UMR 7266 CNRS-Université de La Rochelle, 2 rue Olympe de Gouges, 17000 La Rochelle, France
\end{abstract}

\begin{abstract}
Similarity or dissimilarity between 2 individuals that have formed a pair to breed can occur in morphology, behaviour and diet. Such patterns influence partners' cooperation when rearing their offspring, consequently influencing reproductive success. They may confer different benefits, depending on species and contexts. However, the extent to which breeding partners are more similar in morphology, behaviour, and diet is poorly documented. Furthermore, the relationship between behavioural consistency and mate choice is particularly poorly understood. To investigate these issues, Kerguelen shags Phalacrocorax verrucosus, which are monogamous with high mate fidelity across years, were studied. Partners were equipped with GPS and diving behaviour loggers. Feather and blood samples were analysed for stable isotopes $\left(\delta^{13} C_{\text {, a proxy of }}\right.$ foraging habitat, and $\delta^{15} \mathrm{~N}$, a proxy of diet/trophic position). Generalized linear mixed effects models and permutation tests were used to investigate pair similarity in morphology, foraging behaviour, behavioural consistency, overlap in foraging areas, and diets/foraging habitats. Mates were found not to exhibit size-assortative mating, but were more similar in foraging behaviour. They did not show assortative or disassortative mating based on foraging behavioural consistency. Furthermore, they followed more similar bearings and overlapped more in foraging areas. In accordance with this, partners were more similar in $\delta^{15} \mathrm{~N}$. Given the lack of assortative mating by morphology, the similarity in behaviour could be due to individuals selecting mates with similar foraging abilities, linked with individual quality, and/or subsequently using information gained from their partners' foraging strategies (e.g. local enhancement). This could help breeding pairs increase their foraging efficiency and reproductive success.
\end{abstract}

KEY WORDS: Pair similarity $\cdot$ Mate choice $\cdot$ Kerguelen shags $\cdot$ Spatial use $\cdot$ Diving behaviour Diet $\cdot$ Stable isotopes

\section{INTRODUCTION}

Similarity in behavioural traits within breeding pairs can have important, long-lasting effects on reproductive success and fitness in species with biparental care, probably because of reduced sexual conflict

\footnotetext{
*Corresponding author: elodie.camprasse@gmail.com
}

over the provision of parental investment associated with choosing a partner with compatible provisioning rules (Schuett et al. 2011). This allows mates to enhance their cooperation and coordination in the provision of care, essential in species with biparental care and associated with improved reproductive per-

() The authors 2017. Open Access under Creative Commons by Attribution Licence. Use, distribution and reproduction are unrestricted. Authors and original publication must be credited. 
formance, including offspring growth and survival (Spoon et al. 2006, Schuett et al. 2010, 2011, Rangassamy et al. 2015). Disassortative mating with respect to the partners' recognition cues may, however, lead to reduced inbreeding (Holman et al. 2013). In terms of personality, disassortative mating can lead to the production of offspring of intermediate personality, associated with lower variance in survival in the long-term and higher life expectancy (Dingemanse et al. 2004, Schuett et al. 2010). Risk partitioning may increase the fitness of both partners; while one parent could adopt a risk-averse strategy to provide enough food to ensure that the chicks reach fledging, the other partner might provide the extra bulk for improved post-fledging survival through a riskprone strategy (Elliott et al. 2010). In contrast, combinations of dissimilar behavioural traits within pairs could result in unstable and disharmonious conditions, generating high stress levels that have the potential to negatively influence reproduction (Von Holst 1998, Rangassamy et al. 2015). In general, the extent of mate similarity in behaviour is poorly investigated (Schuett et al. 2010).

Empirical evidence suggests that mate similarity or dissimilarity confer different advantages and are selected for in different species or environmental conditions in a non-mutually exclusive way (Dingemanse et al. 2004, Schuett et al. 2010). In the dumpling squid Euprymna tasmanica, mates showing similar levels of boldness had higher probabilities of reproducing successfully, which might result from either behavioural mate preference or genetic compatibility between partners (Sinn et al. 2006). Similarly, in some bird species, highly behaviourally compatible pair members had higher reproductive success potentially as a result of better cooperation of individuals of similar behavioural traits (Both et al. 2005, Spoon et al. 2006). In contrast, thick-billed murre Uria lomvia pairs exhibited a higher reproductive success when they were constituted of one risk-averse and one risk-prone partner (Elliott et al. 2010). Similarly, in animals with distinct foraging territories such as raptors, overall feeding rates become higher when mates adopt different foraging strategies (Andersson \& Norberg 1981).

Behavioural consistency could also be used to assess the quality of potential mates, and, therefore, influence mate choice (Byers 2007, Botero et al. 2009, de Kort et al. 2009). Consistency in behaviour can signal predictability and, as such, can provide benefits to partners in many aspects of their social life (Schuett et al. 2010). Consistency could also be an indicator of quality and it has been suggested that consistency could be generated by sexual selection if individuals tend to preferentially choose mates that are consistent or individuals outperform competitors when they are consistent (Dall et al. 2004). Assortative mating in terms of behavioural consistency could be important to enhance behavioural coordination within breeding pairs, leading to increased reproductive success (Spoon et al. 2006). In contrast, in cases in which pairs constituted of a risk-prone and a risk-averse mate have a better reproductive success, disassortative mating by behavioural consistency might be preferable; risk-prone individuals might indeed be more inclined to explore new environments (Dingemanse et al. 2003, Bremner-Harrison et al. 2004), and therefore exhibit lower behavioural consistency, compared to their risk-averse mates. Data on assortment in terms of behavioural consistency is lacking, but studies in zebra finches Taeniopygia guttata suggest that pairs comprised of partners that differ in behavioural consistency raise offspring in poorer condition (Schuett et al. 2010, 2011).

Mate similarity or dissimilarity can also be influenced by sexual dimorphism and size-assortative mating. Sexual size dimorphism is widespread in animal taxa, and species exhibiting dimorphism are known to exhibit differences in behaviour and diet (Andersson \& Norberg 1981, Camilleri \& Shine 1990, Magurran \& Garcia 2000, Marcelli et al. 2003, Isaac 2005, Weimerskirch et al. 2006). For example, male and female European polecats Mustela putorius forage at different times of the day (Marcelli et al. 2003) and some snake species exhibit dimorphism leading to dietary divergence between males and females (Camilleri \& Shine 1990). Hence, sexual dimorphism would be expected to influence mate similarity and lead to a higher mate dissimilarity in behaviour and/or diet within dimorphic species (Andersson \& Norberg 1981, Elliott et al. 2010) in comparison to monomorphic species. However, when size-assortative mating occurs, such dissimilarity is likely to be reduced as mates are then more similar in morphology and, therefore, expected to be more similar in behaviour as well in comparison to non-mated individuals. The interplay between dimorphism, sizeassortative mating, and mate similarity in behaviour and diet has rarely been investigated.

Seabirds are generally socially monogamous, exhibit biparental care, and show high mate fidelity (Bried \& Jouventin 2002). As such, seabird partners establish specific foraging strategies in order to enhance their reproductive success through, for example, better coordination of provisioning behaviour (Davis 1988, Shoji et al. 2011, Thiebot et al. 2015). Despite the potentially long-lasting and important 
consequences of pair similarity on reproductive success, only 2 studies, to the best of our knowledge, have focused on identifying pair similarity in the diet and behaviour of seabirds. They showed that partners do not necessarily display similar food preferences (Harris et al. 2016), and that similarity in partners' diets can lead to a decline in chick growth rates and fledglings produced (Watanuki 1992). To fill these knowledge gaps, testing the pair similarity in key traits affecting offspring provisioning and condition, such as in foraging metrics and behavioural consistency during the breeding season, is needed, particularly in dimorphic species in which males and females are expected to differ in behaviour and diet.

Kerguelen shags Phalacrocorax verrucosus are suitable for investigations of relationships in the behaviour, consistency in foraging and diet of partners within a breeding pair. Individuals exhibit strong specialisation in such traits that can be maintained over the long-term, regardless of their sex, and therefore could be used by individuals to evaluate the quality of potential mates (Bearhop et al. 2006, Cook et al. 2006, Camprasse et al. 2017a). They are long-lived, resident, and benthic foraging seabirds, and individuals repetitively exploit the same foraging areas (Camprasse et al. 2017a). In addition, both parents often exhibit high nest fidelity, mate retention, and share incubation and chick-rearing duties (Aebischer et al. 1995, Sapoznikow \& Quintana 2008, C. A. Bost pers. obs.). Kerguelen shags exhibit sexual dimorphism, with males being larger and heavier than females, as well as specialisations in feeding times, with females foraging in the morning and males foraging in the afternoon (Cook et al. 2013); these patterns might be expected to lead to mates exhibiting differences in behaviour, which makes Kerguelen shags interesting models to investigate the interplay between sexual dimorphism, size-assortative mating, and pair similarity.

In the present study, the similarity in foraging behaviour and morphology within pairs of Kerguelen shags was examined through the use of morphometric measurements and the combination of stable isotope dietary analysis and bio-logging techniques. Our aim was to determine whether (1) individuals exhibit size-assortative or -disassortative mating; (2) the foraging behaviour of partners was more or less similar compared to non-mated birds; (3) the consistency in foraging behaviour of partners was more or less similar compared to non-mated birds; (4) partners overlapped more or less than non-mated birds in foraging locations; and (5) partners exhibited more similar or dissimilar diets/foraging habitats compared to non-mated birds.

\section{MATERIALS AND METHODS}

\section{Instrumentation}

Field work was conducted at Pointe Suzanne $\left(49^{\circ} 26^{\prime} \mathrm{S}, 70^{\circ} 26^{\prime} \mathrm{E}\right)$, Kerguelen Island, southern Indian Ocean, during the $2014 / 2015$ breeding season. Sampling occurred during 2 sessions. First, a total of 20 Kerguelen shag Phalacrocorax verrucosus individuals (both partners from 10 nests) were equipped with GPS data loggers (I-gotU GT120, Mobile Action; $44.5 \times 28.5 \times 13 \mathrm{~mm}, 12 \mathrm{~g}$ in air corresponding to ca. $0.5 \%$ of mean body mass) for 3 to $6 \mathrm{~d}$ at the end of the incubation/early chick-rearing period (26 November to 10 December, hereafter 'incubation/early chickrearing'), when chicks were no older than 1 wk. During this session, nest checks every 2 or $3 \mathrm{~d}$ allowed us to determine the age of the chicks. Second, a total of 22 birds (both partners from 11 nests, including 6 new nests and 5 nests used during the first deployment session) were equipped for 3 to $12 \mathrm{~d}$ during the late chick-rearing period (6 to 18 January, hereafter 'late chick-rearing'), of which the 10 previously sampled birds were deployed with GPS data loggers while the remaining 12 individuals were equipped both with GPS data loggers and time-depth recorders (TDRs, LAT1800S, Lotek Wireless; $36 \times 11 \times 7.2 \mathrm{~mm}$, $4.8 \mathrm{~g}$ in air corresponding to ca. $0.2 \%$ of mean body mass). During this second session, chicks were ca. 30 to $40 \mathrm{~d}$ old, except for one pair that had a chick ca. 10 to $15 \mathrm{~d}$ old. Monitoring of the nests could not be conducted at all times but a high proportion of observed change-overs $(75.8 \%)$ occurred after females came back from their morning trips, after which they tended to stay at the colony for the day (E. C. M. Camprasse pers. obs.), and therefore chicks were still in the presence of a parent most of the time. All but one brood had a single chick at deployment. This unique brood, however, lost their second chick immediately after deployment. While no quantitative data was collected on the rest of the population, this low number of chicks per brood was a general pattern within the colony compared to the maximum of 3 chicks that Kerguelen shag pairs can raise during a single breeding season. In conjunction with poor breeding success in sympatrically breeding Gentoo penguins at the time of the study, such a pattern seems indicative of unfavourable environmental conditions (Camprasse et al. 2017b, this Theme Section).

Individuals were captured at the colony using a noose attached to a fishing pole, weighed in a cloth bag using a suspension scale $( \pm 25 \mathrm{~g}$, Pesola), and banded with an individually numbered coloured 
plastic ring on one leg and an individually numbered metal ring on the other leg for identification. The data loggers, encased in heat-shrink plastic for waterproofing, were attached to the back feathers using waterproof tape (Tesa 4651) and cyanoacrylate glue (Loctite 401). Handling times ranged from 15 to 20 min during which the bird's head was covered with a hood to reduce stress. Females tended to forage in the morning while males foraged mostly during the afternoon (Cook et al. 2013). Whenever possible, we took advantage of this difference in schedule to deploy data loggers before the birds left the colony.

Individuals were gently recaptured 3 to $18 \mathrm{~d}$ later as previously described. The data loggers were removed, individuals were weighed again, and morphometric measurements (bill length, bill width, head length, wing length, and tarsus length) were taken using a vernier caliper $( \pm 0.05 \mathrm{~mm})$, or ruler $( \pm 1 \mathrm{~mm})$ (except for one bird for which we obtained mass but not morphometric measurements). In addition, 3 to 6 dorsal dark contour feathers were plucked and a blood sample (0.5 to $1.5 \mathrm{ml})$ was collected by venipuncture of a tarsal vein.

GPS loggers were programmed to sample position at $1 \mathrm{~min}$ intervals during incubation/early chickrearing and at 2 min intervals during the chickrearing period. The TDR units were set to record depth at $1 \mathrm{~s}$ intervals.

GPS data were obtained for only 17 of the 20 individuals equipped during the first round of deployments due to logger failure, resulting in 7 pairs with spatial data for both partners at this stage. During the second deployment session, spatial data were obtained for 21 out of 22 individuals, and thus 10 pairs had complete data for both members. Of these 10 pairs, 6 pairs were from new nests and 4 pairs were from those that were deployed with loggers during incubation; of these 4,2 had complete data from the first deployment session. This resulted in a total number of 15 different pairs with data on both members. For 13 of those pairs, both members had data on more than one trip and could be used to evaluate consistency in spatial use.

\section{Isotopic analyses}

The measurement of ratios of stable isotopes of carbon $\left({ }^{13} \mathrm{C} /{ }^{12} \mathrm{C}\right)$ and nitrogen $\left({ }^{15} \mathrm{~N} /{ }^{14} \mathrm{~N}\right)$ is a powerful tool to investigate the food and feeding ecology of consumers (Cherel et al. 2005a). More specifically, $\delta^{13} \mathrm{C}$ and $\delta^{15} \mathrm{~N}$ values of seabirds are considered to be proxies of their foraging habitats and diets/trophic position, respectively (Cherel \& Hobson 2007). Overall, $\delta^{13} \mathrm{C}$ values decrease along a latitudinal gradient (Cherel \& Hobson 2007, Jaeger et al. 2010) and $\delta^{15} \mathrm{~N}$ values increase with trophic level (Cherel et al. 2010). $\delta^{13} \mathrm{C}$ enrichment also occurs for inshore or benthic species as opposed to offshore or pelagic ones (Hobson et al. 1994). Isotopic values (details in Cherel et al. 2008) were measured on whole blood (hereafter 'blood') and contour feathers (hereafter 'feathers') of the studied shags. The rationale is that the 2 complementary tissues integrate different periods of information. Blood is a metabolically active tissue that covers a period of weeks before sampling, whereas feathers, a metabolically inert tissue, reflect the foraging ecology at the time they were grown. In other species from the blue-eyed shag complex, such as the Antarctic shag P. bransfieldensis, contour feathers are replaced in March, immediately after breeding (Bernstein \& Maxson 1981). Here, blood and feathers collected during the breeding period reflect the breeding period itself and the previous post-breeding moulting period that took place almost 1 yr before the study, respectively.

In the laboratory, blood samples were freeze-dried and powdered. Lipid extraction was not necessary as the C:N mass ratio was $<3.5$ for all blood samples (Cherel et al. 2005b). A single contour feather per bird was cleaned of surface lipids and contaminants using a 2:1 chloroform:methanol bath, air-dried, and cut into small pieces. Nitrogen and carbon isotopic ratios were measured with a continuous flow-isotope ratio mass spectrometer (Thermo Scientific Delta V Advantage) coupled to an elemental analyser (Thermo Scientific Flash EA 1112). Results are presented in the usual $\delta$ notation relative to Vienna PeeDee Belemnite for carbon and atmospheric $\mathrm{N}_{2}$ for nitrogen. Replicate measurements of internal laboratory standards (acetanilide and peptone) indicated measurement errors $<0.15 \%$ for both $\delta^{13} \mathrm{C}$ and $\delta^{15} \mathrm{~N}$. Isotopic values were obtained for both members of 15 pairs.

\section{Data processing}

All data processing and analyses were conducted in the R Statistical Environment, version 3.2 (R Core Team 2015). GPS records were visually inspected and individual trips were determined. The diveMove package (Luque 2007) was used to apply a speed filter on the GPS data to remove erroneous locations and obtain summaries of diving metrics from TDR records (only dives $>1 \mathrm{~m}$ were considered in analyses). As dive depths (i.e. depths at the deepest part of 
a dive) were not normally distributed within individuals, the mode of dive depths instead of the mean was recorded for each trip. The means of dive durations and the sum of vertical distance travelled per trip were also calculated. These values were used to calculate a mean of means (or mean of modes) and coefficients of variation (CVs) across foraging trips for each individual. The packages trip (Sumner 2013) and $s p$ (Pebesma \& Bivand 2015) were used to obtain foraging metrics for each trip (bearings, total distances travelled, maximum distances and trip durations). Bearing for each trip was calculated as the angle between the colony and the most distal point of the tracks and standard deviation in bearing was calculated for each individual using the circular package (Lund \& Agostinelli 2011). Means and CVs for all metrics were obtained per individual and per stage, except bearings, for which SD was calculated.

Kernel home ranges for each trip of each individual were determined in the adehabitatHR package (Calenge 2006). Because Kerguelen shags tend to fly to their foraging grounds and dive predominantly at the most distal part of their trip (Camprasse et al. 2017a), and because we wanted to know whether sexes or partners forage in the same locations, only core foraging area (50\% home range) was calculated. Each trip for each male within each breeding stage was compared to each trip for each female sampled within the same stage by calculating the overlap in core foraging area between these 2 trips with the 'kerneloverlap' function in the adehabitatHR package using the Bhattacharyya's affinity (Fieberg \& Kochanny 2005).

An index of spatial use consistency was calculated for each individual within each stage. For each pair of trips within a deployment, a kernel overlap was calculated (e.g. for a bird with 3 trips, 3 overlap values were obtained, between Trip 1 and Trip 2, Trip 2 and Trip 3 and Trip 1 and Trip 3), as described above for the overlap between males and females. The average of these numbers was obtained and used as an index of consistency for each individual within each stage.

\section{Data analyses}

Linear mixed effects models were used to confirm sexual dimorphism in mass in study birds, as mass data was obtained both at incubation/early chickrearing and in late chick-rearing for some birds, and always both at deployment and retrieval. Dimorphism in size was checked using only 1 of the mor- phometrics obtained (tarsus length, as the other ones were correlated using a linear regression) as single measurements were obtained. When looking at pair similarity, 2 kinds of analyses were run depending on the structure of the data: when a single observation per individual was available, permutation tests were run, and when multiple observations were available (i.e. one observation per trip on multiple consecutive trips), generalized linear mixed models (GLMMs) with crossed random effects were used. p-values $<0.05$ were considered significant for all tests. Specifically, a significant $\mathrm{p}$-value for the permutation tests or binomial GLMM meant that partners were more similar or dissimilar than expected by chance and summarizing the data for 'true pairs' and 'false pairs' gave the direction of the effect.

To investigate any potential correlations in morphology in partners, a principal component analysis (PCA) was run on masses at deployment and retrieval and on the 5 body measurements. The Euclidean distances for all possible combinations of males and females were calculated from the scores obtained thanks to the PCA for each individual. Euclidean distances were used as a way to examine the pattern of similarities in the body size and mass of individuals in the sample (Wojczulanis-Jakubas et al. 2011). Permutation tests were carried out in the permute package (Simpson 2014) on the matrix containing the Euclidean distances and whether they came from actual paired individuals (value of 1 ) or not (value of 0) with the null hypothesis being that partners were not more similar than expected by chance. Permutation tests (10 000 iterations) randomly assigned each Euclidean distance to a type of pairing. Permutational p-values were used and they are defined as the proportion of randomized values as extreme or more extreme than the observed value (Manly 1991).

The 3 dive-level variables that we extracted from the TDR data (dive duration, maximum depth, and vertical distance) were correlated, so we only considered maximum depth in the analysis (maximum depths and trip duration: Spearman's rho $=0.93, \mathrm{p}<$ 0.0001 , maximum depths and sums of vertical distances: Spearman's rho $=0.36, p=0.05$ ). Maximum depths were used in modelling as representative of habitat selection in the vertical dimension. The absolute values of the differences in maximum depth between males and females were used to investigate the similarity or dissimilarity between paired females and males within the chick-rearing stage, because we were not specifically interested in which sex had the higher value, but just in the distance between them. We estimated the probability of pairs constitut- 
ing true pairs using logistic regression in a generalized linear mixed-effects modelling framework ('glmer' in the lme4 package (Bates et al. 2011), with a binomial distribution and logit link). Best models were selected based on their Akaike information criteria. For the diving behaviour model, differences between males and females in maximum dive depths were used as explanatory variables, while the individual bird ID was used as a crossed random effect with males crossed with females of each possible pair (with 1 being the 'true pair' and 0 being a 'false pair'). Total distance travelled, maximum distance and trip duration were highly correlated (total distance and trip duration: Spearman's rho $=0.60, \mathrm{p}<0.0001$, total distance and maximum distance: Spearman's rho = 0.96, $\mathrm{p}<0.0001$ ). Therefore, only total distance travelled was included in the analysis of pair similarity or dissimilarity in spatial use. As such, to investigate whether partners exhibited a similar spatial use, a model similar to the one described above included the absolute values of the differences in total distances between males and females for each possible pair and for each stage. In the final model, these differences, stage and their interaction were used as explanatory variables.

To determine if partners were more similar or dissimilar than non-mated birds in consistency, classical multidimensional scaling was applied and the Euclidean distances for all possible combinations of males and females were calculated separately for each stage. Pairing was indicated (with 1 being 'true pair' and 0 being 'false pair'). Permutation tests were performed and permutational p-values were obtained, as described above, for dive behaviour consistency and spatial use consistency, respectively. For the former, Euclidean distances were calculated from the coefficients of variation in maximum depth, dive duration and sum of vertical distance travelled; in this case, all 3 variables were used as their coefficients of variation were not correlated. For the latter, the variables included in the calculation of the Euclidean distances were the following, uncorrelated measures of consistency: index of spatial use consistency, standard deviations in bearing, and coefficients of variation in trip duration, total and maximum distances.

To examine whether partners of the same nest overlapped more or less than birds from different nests, 2 tests were run: one with bearings and one with the kernel overlap values. In both cases, the probability of pairs constituting true pairs was analysed by logistic regression using a generalized linear mixed-effects model (GLMM with a binomial distribution and logit link). The absolute values of the differences between bearings in true and randomised pairs, and the overlap values between males' and females' trips, stage, and their interaction were used as explanatory variables in 2 different models, while individual bird ID was used as a crossed random effect with males crossed with females of each possible pairing.

To investigate whether partners had a more similar or dissimilar diet/trophic level compared to nonmated birds, the Euclidean distances for all possible combinations of males and females were calculated, separately for each stage, either for $\delta^{13} \mathrm{C}$ and $\delta^{15} \mathrm{~N}$ values combined, for $\delta^{13} \mathrm{C}$ values only, or for $\delta^{15} \mathrm{~N}$ values only, in blood and feathers. Permutation tests were carried out on the matrices containing the Euclidean distances and whether they came from actual paired individuals.

\section{RESULTS}

Equipped Kerguelen shags Phalacrocorax verrucosus were sexually dimorphic (Table 1). Overall, males were (mean \pm SD) $0.29 \pm 0.06 \mathrm{~kg}$ heavier $\left(t_{30}=4.60, \mathrm{p}<0.001\right)$ and had tarsus lengths $2.60 \pm$ $0.65 \mathrm{~mm}\left(t_{29}=3.97, \mathrm{p}<0.001\right)$ greater than females. Partners were not more similar or dissimilar to each other in morphological traits and body mass than expected by chance. Indeed, permutation tests indicated no significant differences between paired individuals and the rest of the individuals sampled (permutational $\mathrm{p}=0.18$ ).

Diving data were obtained for all 12 birds equipped with TDR during late chick-rearing. Males tended to dive deeper and be more variable in dive depths than females (Fig. 1, Table S1 in the Supplement at www.int-res.com/articles/suppl/m578p183_supp.pdf) as shown in Camprasse et al. (2017a). The difference

Table 1. Morphometric measurements (mean $\pm \mathrm{SD}_{\text {; }}$ min. - max.) of all Kerguelen shags equipped at the Pointe Suzanne colony, Kerguelen Islands

\begin{tabular}{|lcc|}
\hline Measurement & Females $(\mathrm{n}=14)$ & Males $(\mathrm{n}=15)$ \\
\hline Body mass $(\mathrm{kg})$ & $2.1 \pm 0.2(1.9-2.6)$ & $2.4 \pm 0.2(2.0-2.8)$ \\
Head length $(\mathrm{mm})$ & $132.1 \pm 6.9(124.5-149.0)$ & $133.6 \pm 4.4(125.5-142.0)$ \\
Tarsus length (mm) & $63.6 \pm 1.5(60.9-66.2)$ & $66.1 \pm 2.9(61.4-69.2)$ \\
Beak width $(\mathrm{mm})$ & $13.2 \pm 1.4(11.9-16.9)$ & $14.0 \pm 1.0(12.7-15.7)$ \\
Culmen length (mm) & $50.3 \pm 1.7(47.3-52.6)$ & $52.4 \pm 3.8(40.9-56.6)$ \\
Wing length (mm) & $272.0 \pm 7.4(252.0-282.0)$ & $278.9 \pm 27.9(183.0-295.0)$ \\
\hline
\end{tabular}


in maximum depth between mates was lower than for nonmated birds (binomial GLMM: $\chi^{2}=68.34$, df $\left.=1, \mathrm{p}<0.0001\right)$; on average mates differed in depths by $17.1 \pm 0.9 \mathrm{~m}$, as opposed to $27.6 \pm 0.5 \mathrm{~m}$ in nonmated birds. Males and females did not differ in trip metrics, including trip durations, maximum distances, total distances, and bearings (Tables 2 \& S2). Partners were more similar in spatial use than expected by chance. Indeed, the differences in total distances travelled were smaller in true pairs compared to randomised pairs (binomial GLMM: $\chi^{2}=26.14$, df $=1, \mathrm{p}<0.0001$ ). Further, there was a significant effect of stage on the differences in total distances (binomial GLMM: $\chi^{2}=$ 7.74, df $=1, \mathrm{p}=0.005$ ), and a significant interaction between pairing and stage (binomial GLMM: $\chi^{2}=$ 24.90, df $=1, \mathrm{p}<0.0001)$. Specifically, mates differed in total distances travelled by $14.95 \pm 0.80 \mathrm{~km}$ at incubation/early chick-rearing, and $16.38 \pm 0.71 \mathrm{~km}$ in late chick-rearing; in contrast, the differences in total distances for non-paired birds did not significantly change between incubation/early chick-rearing (19.80 $\pm 0.36 \mathrm{~km})$ and in late chick-rearing $(19.61 \pm 0.25 \mathrm{~km})$.
Within pairs, no specific pattern was observed in terms of the consistency of males and females, both in terms of diving behaviour (Fig. 1) and spatial use. Paired birds were not more similar or dissimilar in consistency in maximum depth, dive duration, and sum of vertical distance (permutational $p=0.14$ ). Lastly, paired birds were not more similar or dissimilar in spatial use consistency (consistency index, SD in bearing, $\mathrm{CV}$ in maximum distance, total distance, and trip duration) compared to non-paired birds (permutational $\mathrm{p}=0.51$ ).

Males always departed after their mates came back from their morning foraging trips during incubation/early chick-rearing, and did so $75.8 \%$ of the time during late chick-rearing. Within pairs, males and

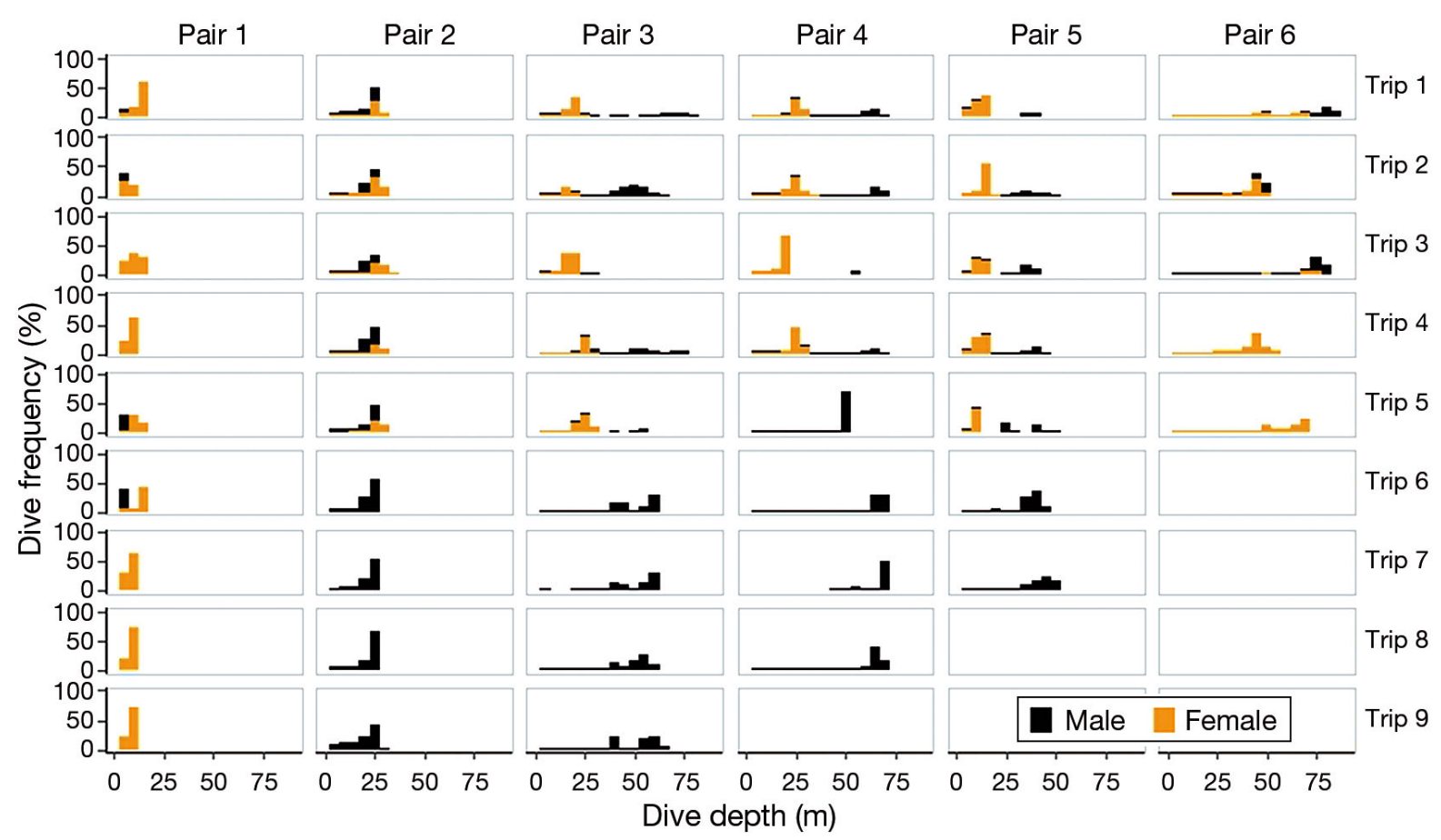

Fig. 1. Dive distributions in relation to dive depth for pairs of Kerguelen shags Phalacrocorax verrucosus equipped at the Pointe Suzanne colony, Kerguelen Islands 

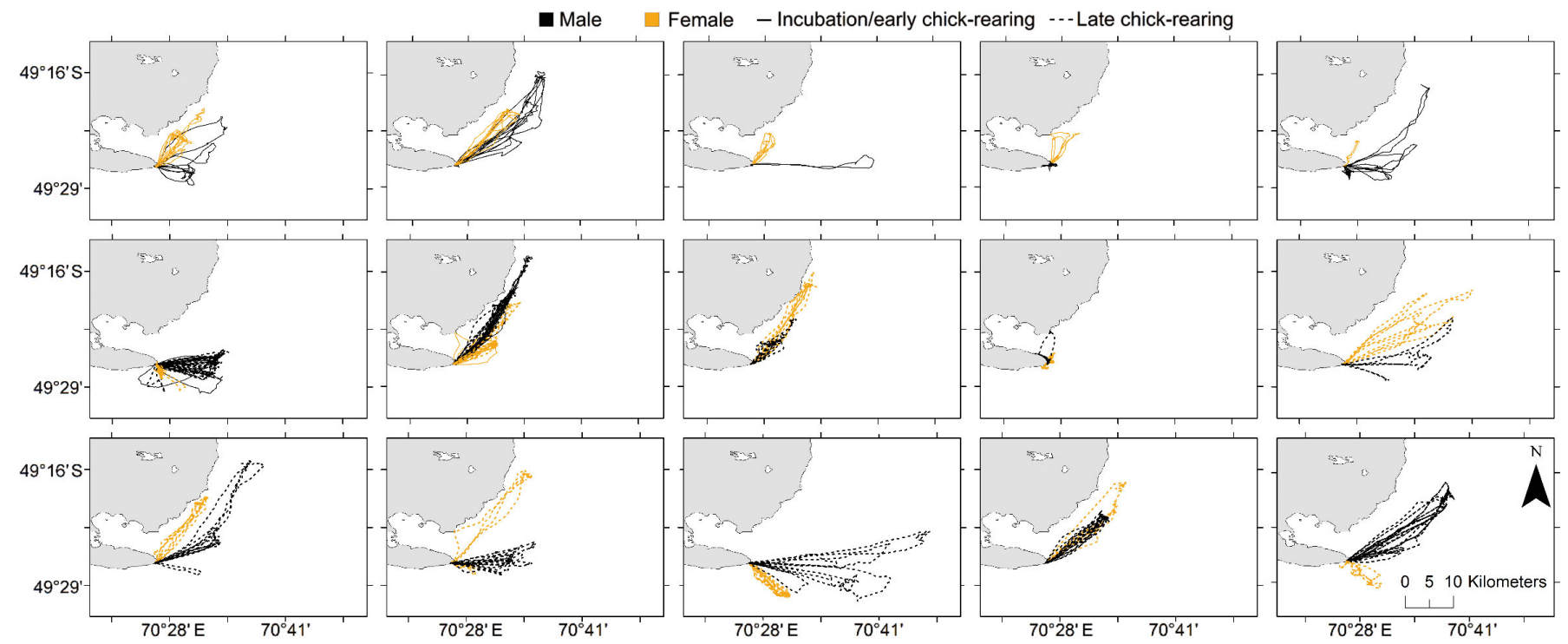

Fig. 2. Consecutive GPS tracks for all pairs of Kerguelen shags Phalacrocorax verrucosus equipped at the Pointe Suzanne colony, Kerguelen Islands

females followed similar bearings to their foraging areas (binomial GLMM: $\chi^{2}=187.14$, df $=1, \mathrm{p}<$ 0.0001) (Fig. 2) and there was a significant effect of stage on differences in bearings (binomial GLMM: $\left.\chi^{2}=32.36, \mathrm{df}=1, \mathrm{p}<0.0001\right)$, but no interaction between stage and the differences in bearings (binomial GLMM: $\chi^{2}=2.60, \mathrm{df}=1, \mathrm{p}=0.11$ ). Mates differed in bearings by $41.8 \pm 2.9^{\circ}$ at incubation/early chick-rearing and $51.98 \pm 2.2^{\circ}$ in late chick-rearing; in contrast, the differences in bearings for non-paired birds were $55.7 \pm 1.0^{\circ}$ at incubation/early chick-rearing and $70.68 \pm 0.8^{\circ}$ in late chick-rearing. Overall, paired birds overlapped significantly more than nonpaired birds (binomial GLMM: $\chi^{2}=159.05, \mathrm{df}=1, \mathrm{p}<$ 0.0001 ) and overlap was not affected by stage (binomial GLMM: $\chi^{2}=1.60, \mathrm{df}=1, \mathrm{p}=0.21$ ). The mean overlap was $14.5 \pm 0.6 \%$ for paired birds and $9.8 \pm$ $0.2 \%$ for non-paired birds.

There were large inter-individual differences in both $\delta^{13} \mathrm{C}$ and $\delta^{15} \mathrm{~N}$ values in blood of the sampled Kerguelen shags (difference: 5.5 and $3.6 \%$, respectively) (Table S3, Fig. S1). Blood $\delta^{13} \mathrm{C}$ values ranged from -19.97 to -14.44 and $\delta^{15} \mathrm{~N}$ values ranged from 12.88 to 16.68 . There were no differences in blood isotopic values between sexes, thus confirming that males and females had similar foraging habitats $\left(\delta^{13} \mathrm{C}\right)$ and diet/trophic levels $\left(\delta^{15} \mathrm{~N}\right)$ during breeding. When taking into account both blood $\delta^{13} \mathrm{C}$ and $\delta^{15} \mathrm{~N}$ values, partners were not more similar or dissimilar than expected by chance for both stages (permutational $p=0.43$ and $p=0.31$, at incubation/early chick-rearing and late chick-rearing, respectively). Blood $\delta^{13} \mathrm{C}$ values of paired birds were not more similar or dissimilar than expected by chance (permutational $\mathrm{p}=0.86$ and $\mathrm{p}=0.74$, at incubation/ early chick-rearing and late chick-rearing, respectively). However, blood $\delta^{15} \mathrm{~N}$ values of paired birds were closer than expected by chance (permutational $p=0.04$ and $p=0.01$, at incubation/early chickrearing and late chick-rearing, respectively). Indeed, in contrast to blood $\delta^{13} \mathrm{C}$ values, male blood $\delta^{15} \mathrm{~N}$ values were positively linearly correlated to female $\delta^{15} \mathrm{~N}$ values (Fig. 3). As with blood, there were large interindividual differences in feathers in both $\delta^{13} \mathrm{C}$ and $\delta^{15} \mathrm{~N}$ values of the sampled Kerguelen shags (difference: 6.7 and $3.9 \%$, respectively), with $\delta^{13} \mathrm{C}$ values ranging from -20.06 to -13.32 and $\delta^{15} \mathrm{~N}$ values ranging from 13.47 to 17.41 . Partners did not have more
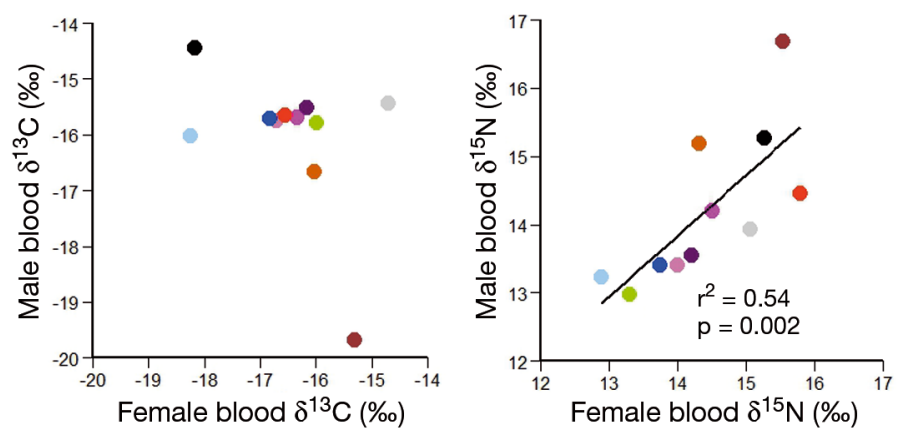

Fig. 3. Correlations in blood $\delta^{13} \mathrm{C}$ and $\delta^{15} \mathrm{~N}$ values of partners of Kerguelen shags Phalacrocorax verrucosus (partners are indicated by shared colours, $\mathrm{n}=11$ pairs) sampled at the Pointe Suzanne colony, Kerguelen Islands; $\delta^{15} \mathrm{~N} \boldsymbol{o}^{7}=0.89 \cdot \delta^{15} \mathrm{~N}$ o +1.42 
similar feather isotopic values, whether taking into account both $\delta^{13} \mathrm{C}$ and $\delta^{15} \mathrm{~N}$ values, $\delta^{13} \mathrm{C}$ values only, or $\delta^{15} \mathrm{~N}$ values only (permutational $\mathrm{p}=0.59,0.68$, and $\mathrm{p}=0.10$, respectively).

\section{DISCUSSION AND CONCLUSIONS}

In the present study, we investigated the similarity or dissimilarity in the foraging strategies of Kerguelen shag Phalacrocorax verrucosus partners using the complementary approaches of bio-logging and stable isotope analysis. The salient results can be summarized as follows: (1) mates did not show assortative or disassortative mating by either morphometrics or behavioural consistency; (2) they were, however, more similar than expected by chance in foraging behaviour, followed more similar bearings, and overlapped more in foraging areas, and (3) they had more similar diets/similar trophic levels than expected by chance.

Our results confirm previous findings that Kerguelen shags are dimorphic in body size and mass (Cook et al. 2013). Within breeding pairs, males were always heavier and generally structurally larger than their female partners. Such strong sexual dimorphism suggests a differential niche utilization for males and females in the population (Selander 1966, Cook et al. 2013) with the potential to affect pair similarity/dissimilarity in foraging behaviour. Indeed, it is expected that dimorphic mates exhibit a higher dissimilarity compared to monomorphic species, as dimorphic birds can display divergent foraging behaviours based on their morphology (Weimerskirch et al. 2006, Elliott et al. 2010). While Kerguelen shags are dimorphic and exhibit temporal segregation with females foraging mostly in the morning and males mostly in the afternoon, differences in their foraging behaviour and its consistency during the time of this study were shown to be limited (Camprasse et al. 2017a). If sexual dimorphism led males and females to display different foraging strategies, then pair dissimilarity in foraging behaviour would be expected. However, in the present study, differences in foraging behaviour between males and females were limited, so if any pair dissimilarity in foraging behaviour were found, it would be due to different factors.

In the present study, we found no evidence of sizeassortative or -disassortative mating. Although seabirds are known to mate assortatively by size (Wagner 1999, Forero et al. 2001, Helfenstein et al. 2004), structural size is not necessarily enough to explain mate choice (Bried \& Jouventin 2002, Berzins et al.
2009). Indeed, in some cases, assortative mating is based on ornamental traits such as plumage or foot/ beak colour, rather than structural size (Berzins et al. 2009, Nolan et al. 2010). For example, in other Phalacrocorax species, assortative mating with respect to crest size has been shown to occur, with crest size being an indicator of individual condition (Daunt et al. 2003). Alternatively, lack of size-assortative mating has been shown in birds and might happen when a specific trait is sexually selected in one sex only (Murphy 2008), which seems less likely in our study species as it has not been shown in shags. Instead of preferring mates that are similar to themselves, all individuals could also show the same preferences for a trait, especially when it is an honest signal indicative of individual quality (Jones et al. 2008, Schuett et al. 2010).

Despite the sexual dimorphism and the lack of sizeassortative mating that was expected to reduce the degree of behavioural similarity in dimorphic mates, our findings suggest that partners are still more similar than non-mated birds in foraging behaviour. Indeed, mates exhibited smaller differences in dive depths and total distances travelled compared to non-paired birds. This pairing in terms of foraging behaviour might result from an active choice if individuals are able to evaluate potential mates and their quality, as individual quality and foraging parameters have been shown to be linked (Lewis et al. 2006, Lescroël et al. 2010). For example, in common murres Uria aalgae, females of higher quality had increased chick feeding rates and lower trip durations (Lewis et al. 2006). Similar patterns were found in Adélie penguins Pygoscelis adeliae, especially at the end of the breeding season, with poorer breeders diving deeper and making longer trips (Lescroël et al. 2010). Alternatively, this pair similarity could be a consequence of developing similar behaviours after pairing, as a result of communication between mates. Studies investigating the similarity or dissimilarity in behaviour in partners are lacking. Contrasting with our results, imperial shag Phalacrocorax atriceps pairs were shown to be constituted of either both benthic members, both pelagic members, or a mixture of both (Harris et al. 2016). Positive assortment by behaviour has been shown in a few studies in groups such as birds (Both et al. 2005, Schuett et al. 2011), fishes (Budaev et al. 1999), and cephalopods (Sinn et al. 2006). In other contexts, better reproductive outcomes might be associated with the fact that more similar individuals exhibit improved cooperation and coordination, leading, for example, to better provisioning of offspring (Spoon et al. 2006, 
Schuett et al. 2010, Sánchez-Macouzet et al. 2014). This might explain the fact that mates were more similar to each other at incubation/early chick-rearing compared to late chick-rearing, as increased cooperation and coordination is more crucial when mates are incubating or guarding small chicks, as this is the period where parents are most at risk of losing their eggs or chicks through accidental dislodgement, predation, and/or hypothermy (Tveraa et al. 1998, Kober \& Gaston 2003, Catry et al. 2006).

There is some evidence that certain combinations of levels of consistency within pairs can influence their reproductive success, and behavioural consistency might be important for mate choice (Schuett et al. 2010, 2011). An individual might benefit from choosing a partner exhibiting consistent behaviour; for example, it might profit from having a mate showing a consistent level of paternal care or territory defence by avoiding having to constantly re-assess its mate's quality and accordingly adjust its own behaviour (Schuett et al. 2010). Assortative mating by behavioural consistency might be expected when consistency is an indicator of quality and/or predictability in provisioning behaviour and parental care; consistent individuals would then be expected to mate preferentially with consistent individuals, resulting in inconsistent individuals having to mate with each other (Schuett et al. 2010). Furthermore, it might be beneficial to choose a mate with high consistency, associated with the benefits mentioned above, but dissimilar behaviour, linked with the acquisition of more diversified prey items, for example (Watanuki 1992). Risk partitioning can increase fitness in paired individuals, with one partner adopting a risk-averse strategy to provide enough food for chicks to be able to fledge and the other exhibiting a risk-prone strategy to provide the extra bulk for enhanced post-fledgling survival (Elliott et al. 2010). As risk-prone individuals can be more inclined to explore new environments and, therefore, be less consistent in their foraging behaviour (Dingemanse et al. 2003, Bremner-Harrison et al. 2004), disassortative mating by behavioural consistency has the potential to be beneficial in pairs, resulting in improved fitness. Despite these apparent advantages of assortative or disassortative mating by behavioural consistency, no such pattern was observed in Kerguelen shags at our study site. Hence, individuals in the present study might predict their mates' provisioning behaviour based on their similarity in foraging behaviour rather than whether they are similarly consistent or not.

We propose that breeding success is related more to foraging behaviour than consistency in foraging behaviour. For example, poorer breeders might have longer trip durations, associated with longer distances travelled and better breeders might dive deeper (Lewis et al. 2006, Lescroël et al. 2010). The breeding success of pairs (number of fledglings, fledgling mass) in our study could not be determined, however, and we could not determine whether more similar or dissimilar partners in terms of behaviour or behavioural consistency had a higher breeding success. Future studies should aim at incorporating such parameters, as well as investigate the influence of differences in environmental conditions and thus prey availability, on pair similarity. In order to better understand the interplay between pair similarity and environmental conditions, it would be necessary to quantify pair similarity and breeding success in moderate environmental conditions, when among-pair variation is likely to be greatest.

In the present study, partners were found to follow more similar bearings, especially at incubation/early chick-rearing, as well as to overlap significantly more in foraging areas compared to non-mated birds. To the best of our knowledge, such a pattern differs from the only study reporting on mate overlap in spatial use in central-place foragers: imperial shag partners in Argentina did not seem to overlap, although whether partners overlapped more or less than nonpaired birds was not tested (Harris et al. 2016). The results shown in our study suggest that such patterns could result from mate choice and/or could allow birds to reduce time spent searching for food if individuals use information gained regarding their partners' foraging strategies to adopt more efficient tactics (e.g. local enhancement). Indeed, seabirds, including cormorants, are known to be able to use visual, tactile, and olfactive cues from their congeners for more efficient foraging (Ward \& Zahavi 1973, Silverman et al. 2004, Weimerskirch et al. 2010) as are other groups of animals (Galef \& Wigmore 1983, Drapier et al. 2002, White et al. 2008). Furthermore, seabirds are able to use information transfer and depart the colony following the direction from which conspecifics are returning to the colony (Tremblay et al. 2014). A transfer of information between paired birds would be facilitated by the temporal segregation in foraging between males and females, with males cueing on their partners' flight directions as they return from morning foraging trips and follow more similar bearings to their partners compared to other conspecifics (Tremblay et al. 2014). Such similarity not only in bearings but also in foraging areas might also explain why mates tended to dive at more similar depths, as Kerguelen shags are benthic divers. 
The spatial overlap in foraging range shown here could explain why partners tended to consume prey at similar trophic levels. This pattern did not occur outside of the breeding season, when birds are not constrained to come back to the nest and therefore are less likely to gather information from their partners, as judged by the $\delta^{15} \mathrm{~N}$ values of feathers. Assortative mating by diet has been shown to occur in fishes and may reflect either the ability of individuals to evaluate potential mates or a consequence of another preference (e.g. habitat choice, morphology) (Snowberg \& Bolnick 2008, Martin 2013). Therefore, assortative mating by diet could either reflect a preference for partners that have similar diets or be a consequence of other factors after pairing based on other criteria has occurred. As it is unclear how individuals are able to assess prospective mates' diet, it seems more likely that individuals choose mates based on their individual quality, for example via the selection of mates with similar crest sizes (Daunt et al. 2003), and then feed at similar trophic levels as a consequence of exhibiting similar foraging behaviour and prospecting for food in similar areas. In contrast, no correlation was observed within breeding pairs in the plasma $\delta^{15} \mathrm{~N}$ values of imperial shags and all potential combinations of foraging behaviour were found within pairs (both partners were benthic feeders, or pelagic feeders, or the pairs were mixed, Harris et al. 2016). Similarity in food preferences within breeding pairs has also been reported in great skuas Stercorarius skua and slaty-backed gulls Larus schistisagus, in which, in contrast to Kerguelen shags, mates frequently hunt together (Watanuki 1992, Votier et al. 2004). In slaty-backed gulls, the increase in diet overlap between mates corresponded with a decrease in chick growth rates and number of fledglings produced (Watanuki 1992). Therefore, the benefits for partners of foraging on similar prey remain unclear, unless it derives from mates communicating on where to find food for reduced searching time and improved chick provisioning as suggested above.

Other factors could lead to mates being more similar foragers than expected by chance which also affect their ability to rear offspring (Bradley et al. 1995, Jouventin et al. 1999, Ludwig \& Becker 2008); some species exhibit age-specific dietary and spatial segregation, primarily determined by a 'cohort effect' that would lead individuals sharing a common life history to forage preferentially together or to share similar foraging limitations (Pelletier et al. 2014). Lastly, subcolony variation, known to occur even in small colonies, could indirectly influence the overlap in forag- ing areas and the similarity in diet within pairs (Masello et al. 2010, Bogdanova et al. 2014). In those studies, individuals at similar locations within the colony tended to forage in the same direction. In our study, however, birds were equipped within a few meters of each other yet still foraged repeatedly in different directions (Camprasse et al. 2017a).

In conclusion, Kerguelen shags were paired with partners that displayed more similar foraging behaviour, foraging bearings, and overlap in foraging areas and diet trophic level than expected by chance. We suggest that shags may pair with individuals of similar quality (e.g. via selection for individual of similar crest size or other traits signalling quality), resulting in mates having similar foraging abilities. They thus have the potential to forage in areas of similar characteristics. Further exchange of information between mates at change-overs could then lead mates to follow similar bearings and overlap more in foraging areas compared to non-paired birds; in turn, this may lead mates to feed at more similar trophic levels. Investigating the ways in which birds assess mate quality (e.g. in terms of foraging efficiency, ornamentation, condition or experience) is crucial to build on the conclusions of the present study. Collecting data during years of different environmental conditions will also help understand if the patterns highlighted in our study are maintained in years of better food availability. Lastly, more studies are necessary to understand the reproductive consequences of mate similarity and should incorporate, for example, longterm measures of reproductive success (Fraser et al. 2002). Such a step is crucial to understand the repercussions of mate choice in seabirds.

Acknowledgements. This study was approved by the ethics committee of the French Polar Institute (Institut Paul-Emile Victor, IPEV). All animals in this study were cared for in accordance with its guidelines. The authors thank volunteers for their hard work in the field, including Ayala Loisel, Elie Gaget, Florian Orgeret, Joris Laborie, and Martin Delpuech. The authors also acknowledge the help of David Pinaud, Matthew Symonds, Lauren Angel, Marlenne Rodríguez, Katherine Brownlie, and Samantha Patrick for assistance with data processing, statistical analyses, and proofreading. Furthermore, we thank Maud Brault-Favrou and Gaël Guillou from the platform Analyses Isotopique of LIENSs for their contribution to sample preparation and isotopic analysis, respectively. We are grateful to Francis Daunt, the handling editor, and to 3 anonymous reviewers for their detailed and constructive comments throughout the revision process. This work was supported financially and logistically by the French Polar Institute (Institut PaulEmile Victor, IPEV, Program 394 'Oiseaux Plongeurs' conducted by C.A.B.) and the Terres Australes et Antarctiques Françaises. 


\section{LITERATURE CITED}

Aebischer NJ, Potts GR, Coulson JC (1995) Site and mate fidelity of shags Phalacrocorax aristotelis at two British colonies. Ibis 137:19-28

Andersson M, Norberg RÅ (1981) Evolution of reversed sexual size dimorphism and role partitioning among predatory birds, with a size scaling of flight performance. Biol J Linn Soc 15:105-130

Bates D, Maechler M, Bolker B (2011) R package lme4: linear mixed-effects models using S4 classes (version 1.1-6). https://cran.r-project.org/package=lme4

Bearhop S, Phillips RA, McGill R, Cherel Y, Dawson DA, Croxall JP (2006) Stable isotopes indicate sex-specific and long-term individual foraging specialisation in diving seabirds. Mar Ecol Prog Ser 311:157-164

Bernstein NP, Maxson SJ (1981) Notes on moult and seasonably variable characters of the Antarctic blue-eyed shag Phalacrocorax atriceps bransfieldensis. Notornis 28:35-39

Berzins LL, Gilchrist HG, Burness G (2009) No assortative mating based on size in black guillemots breeding in the Canadian Arctic. Waterbirds 32:459-463

Bogdanova MI, Wanless S, Harris MP, Lindström J and others (2014) Among-year and within-population variation in foraging distribution of European shags Phalacrocorax aristotelis over two decades: implications for marine spatial planning. Biol Conserv 170:292-299

Botero CA, Rossman RJ, Caro LM, Stenzler LM, Lovette IJ, de Kort SR, Vehrencamp SL (2009) Syllable type consistency is related to age, social status and reproductive success in the tropical mockingbird. Anim Behav 77: 701-706

Both C, Dingemanse NJ, Drent PJ, Tinbergen JM (2005) Pairs of extreme avian personalities have highest reproductive success. J Anim Ecol 74:667-674

Bradley JS, Wooller RD, Skira IJ (1995) The relationship of pair-bond formation and duration to reproductive success in short-tailed shearwaters Puffinus tenuirostris. J Anim Ecol 64:31-38

Bremner-Harrison S, Prodohl P, Elwood RW (2004) Behavioural trait assessment as a release criterion: boldness predicts early death in a reintroduction programme of captive-bred swift fox (Vulpes velox). Anim Conserv 7: 313-320

Bried J, Jouventin P (2002) Site and mate choice in seabirds: an evolutionary approach. In: Schreiber EA, Burger J (eds) Biology of marine birds. CRC Marine Biology Series. CRC Press, Boca Raton, FL, p 263-305.

Budaev SV, Zworykin DD, Mochek AD (1999) Individual differences in parental care and behaviour profile in the convict cichlid: a correlation study. Anim Behav 58: 195-202

Byers BE (2007) Extrapair paternity in chestnut-sided warblers is correlated with consistent vocal performance. Behav Ecol 18:130-136

* Calenge C (2006) The package 'adehabitat' for the R software: a tool for the analysis of space and habitat use by animals. Ecol Model 197:516-519

Camilleri C, Shine R (1990) Sexual dimorphism and dietary divergence: differences in trophic morphology between male and female snakes. Copeia 649-658

Camprasse ECM, Cherel Y, Arnould JPY, Hoskins AJ, Bost CA (2017a) Combined bio-logging and stable isotopes reveal individual specialisations in a benthic coastal seabird, the Kerguelen shag. PLOS ONE 12:e0172278
Camprasse ECM, Cherel Y, Bustamante P, Arnould JPY, Bost CA (2017b) Intra- and inter-individual variation in the foraging ecology of a generalist subantarctic seabird, the gentoo penguin. Mar Ecol Prog Ser 578:227-242

Catry P, Phillips RA, Forcada J, Croxall JP (2006) Factors affecting the solution of a parental dilemma in albatrosses: at what age should chicks be left unattended? Anim Behav 72:383-391

* Cherel Y, Hobson KA (2007) Geographical variation in carbon stable isotope signatures of marine predators: a tool to investigate their foraging areas in the Southern Ocean. Mar Ecol Prog Ser 329:281-287

Cherel Y, Hobson KA, Bailleul F, Groscolas R (2005a) Nutrition, physiology, and stable isotopes: new information from fasting and molting penguins. Ecology 86:2881-2888

* Cherel Y, Hobson KA, Hassani S (2005b) Isotopic discrimination between food and blood and feathers of captive penguins: implications for dietary studies in the wild. Physiol Biochem Zool 78:106-115

* Cherel Y, Le Corre M, Jaquemet S, Menard F, Richard P, Weimerskirch $H$ (2008) Resource partitioning within a tropical seabird community: new information from stable isotopes. Mar Ecol Prog Ser 366:281-291

Cherel Y, Fontaine C, Richard P, Labatc JP (2010) Isotopic niches and trophic levels of myctophid fishes and their predators in the Southern Ocean. Limnol Oceanogr 55: 324-332

Cook TR, Cherel Y, Tremblay Y (2006) Foraging tactics of chick-rearing Crozet shags: individuals display repetitive activity and diving patterns over time. Polar Biol 29: 562-569

* Cook TR, Lescroël A, Cherel Y, Kato A, Bost CA (2013) Can foraging ecology drive the evolution of body size in a diving endotherm? PLOS ONE 8:e56297

* Dall SR, Houston AI, McNamara JM (2004) The behavioural ecology of personality: consistent individual differences from an adaptive perspective. Ecol Lett 7:734-739

* Daunt F, Monaghan P, Wanless S, Harris MP (2003) Sexual ornament size and breeding performance in female and male European shags Phalacrocorax aristotelis. Ibis 145: $54-60$

Davis LS (1988) Coordination of incubation routines and mate choice in Adélie penguins Pygoscelis adeliae. Auk 105:428-432

de Kort SR, Eldermire ER, Valderrama S, Botero CA, Vehrencamp SL (2009) Trill consistency is an age-related assessment signal in banded wrens. Proc R Soc B 276: 2315-2321

Dingemanse NJ, Both C, Van Noordwijk AJ, Rutten AL, Drent PJ (2003) Natal dispersal and personalities in great tits (Parus major). Proc R Soc B 270:741-747

Dingemanse NJ, Both C, Drent PJ, Tinbergen JM (2004) Fitness consequences of avian personalities in a fluctuating environment. Proc R Soc B 271:847-852

* Drapier M, Chauvin C, Thierry B (2002) Tonkean macaques Macaca tonkeana find food sources from cues conveyed by group-mates. Anim Cogn 5:159-165

* Elliott KH, Gaston AJ, Crump D (2010) Sex-specific behavior by a monomorphic seabird represents risk partitioning. Behav Ecol 21:1024-1032

* Fieberg J, Kochanny CO (2005) Quantifying home-range overlap: the importance of the utilization distribution. J Wildl Manag 69:1346-1359

*Forero MG, Tella JL, Donázar JA, Blanco G, Bertellotti M, Ceballos O (2001) Phenotypic assortative mating and 
within-pair sexual dimorphism and its influence on breeding success and offspring quality in Magellanic penguins. Can J Zool 79:1414-1422

Fraser GS, Jones IL, Hunter FM (2002) Male-female differences in parental care in monogamous crested auklets. Condor 104:413-423

Galef BGJ, Wigmore SW (1983) Transfer of information concerning distant foods: a laboratory investigation of the 'information-centre' hypothesis. Anim Behav 31:748-758

Harris S, Quintana F, Ciancio J, Riccialdelli L, Raya Rey A (2016) Linking foraging behavior and diet in a diving seabird. Mar Ecol 37:419-432

Helfenstein F, Danchin E, Wagner RH (2004) Assortative mating and sexual size dimorphism in black-legged kittiwakes. Waterbirds 27:350-354

Hobson KA, Piatt JF, Pitocchelli J (1994) Using stable isotopes to determine seabird trophic relationships. J Anim Ecol 63:786-798

*Holman L, Van Zweden JS, Linksvayer TA, d'Ettorre P (2013) Crozier's paradox revisited: maintenance of genetic recognition systems by disassortative mating. BMC Evol Biol 13:211

Isaac JL (2005) Potential causes and life-history consequences of sexual size dimorphism in mammals. Mammal Rev 35:101-115

Jaeger A, Lecomte VJ, Weimerskirch H, Richard P, Cherel Y (2010) Seabird satellite tracking validates the use of latitudinal isoscapes to depict predators' foraging areas in the Southern Ocean. Rapid Commun Mass Spectrom 24: 3456-3460

Jones FC, Brown C, Braithwaite VA (2008) Lack of assortative mating between incipient species of stickleback from a hybrid zone. Behaviour 145:463-484

Jouventin P, Lequette B, Dobson FS (1999) Age-related mate choice in the wandering albatross. Anim Behav 57: 1099-1106

Kober K, Gaston AJ (2003) Social interactions among breeding Brünnich's guillemots Uria lomvia suggest constraints in relation to offspring vulnerability. Ibis 145:413-418

* Lescroël A, Ballard G, Toniolo V, Barton KJ, Wilson PR, Lyver POB, Ainley DG (2010) Working less to gain more: When breeding quality relates to foraging efficiency. Ecology 91:2044-2055

Lewis S, Wanless S, Elston DA, Schultz MD and others (2006) Determinants of quality in a long-lived colonial species. J Anim Ecol 75:1304-1312

Ludwig SC, Becker PH (2008) Supply and demand: causes and consequences of assortative mating in common terns Sterna hirundo. Behav Ecol Sociobiol 62:1601-1611

* Lund U, Agostinelli C (2011) 'Circular' in R. R package version 0.4-7, https://cran.r-project.org/package=circular

Luque SP (2007) Diving behaviour analysis in R. R News 7: 8-14

Magurran A, Garcia CM (2000) Sex differences in behaviour as an indirect consequence of mating system. J Fish Biol 57:839-857

Manly BFJ (1991) Randomization and Monte Carlo methods in biology. Chapman \& Hall, London

Marcelli M, Fusillo R, Boitani L (2003) Sexual segregation in the activity patterns of European polecats (Mustela putorius). J Zool (Lond) 261:249-255

Martin CH (2013) Strong assortative mating by diet, color, size, and morphology but limited progress toward sympatric speciation in a classic example: Cameroon crater lake cichlids. Evolution 67:2114-2123
Masello JF, Mundry R, Poisbleau M, Demongin L, Voigt CC, Wikelski M, Quillfeldt P (2010) Diving seabirds share foraging space and time within and among species. Ecosphere 1:art19

Murphy TG (2008) Lack of assortative mating for tail, body size, or condition in the elaborate monomorphic turquoisebrowed motmot Eumomota superciliosa. Auk 125:11-19

Nolan PM, Dobson FS, Nicolaus M, Karels TJ, McGraw KJ, Jouventin P (2010) Mutual mate choice for colorful traits in king penguins. Ethology 116:635-644

* Pebesma E, Bivand R (2015) sp: classes and methods for spatial data. R package version 1.1-1, https://cran.rproject.org/package $=\mathrm{sp}$

* Pelletier L, Chiaradia A, Kato A, Ropert-Coudert Y (2014) Fine-scale spatial age segregation in the limited foraging area of an inshore seabird species, the little penguin. Oecologia 176:399-408

R Core Team (2015) R: A language and environment for statistical computing. R Foundation for Statistical Computing, Vienna

* Rangassamy M, Dalmas M, Féron C, Gouat P, Rödel HG (2015) Similarity of personalities speeds up reproduction in pairs of a monogamous rodent. Anim Behav 103: $7-15$

Sánchez-Macouzet O, Rodríguez C, Drummond H (2014) Better stay together: pair bond duration increases individual fitness independent of age-related variation. Proc R Soc B 281:20132843

Sapoznikow A, Quintana F (2008) Colony and nest site fidelity of the rock shag Phalacrocorax magellanicus. J Ornithol 149:639-642

* Schuett W, Tregenza T, Dall SRX (2010) Sexual selection and animal personality. Biol Rev Camb Philos Soc 85: $217-246$

* Schuett W, Dall SRX, Royle NJ (2011) Pairs of zebra finches with similar 'personalities' make better parents. Anim Behav 81:609-618

Selander RK (1966) Sexual dimorphism and differential niche utilization in birds. Condor 68:113-151

* Shoji A, Elliott KH, Aris-Brosou S, Crump D, Gaston AJ (2011) Incubation patterns in a central-place forager affect lifetime reproductive success: scaling of patterns from a foraging bout to a lifetime. PLOS ONE 6:e17760

Silverman ED, Veit RR, Nevitt GA (2004) Nearest neighbors as foraging cues: information transfer in a patchy environment. Mar Ecol Prog Ser 277:25-35

Simpson GL (2014) R package permute: functions for generating restricted permutations of data (version 0.8-3). http://CRAN.R-project.org/package=permute

* Sinn DL, Apiolaza LA, Moltschaniwskyj NA (2006) Heritability and fitness-related consequences of squid personality traits. J Evol Biol 19:1437-1447

* Snowberg LK, Bolnick DI (2008) Assortative mating by diet in a phenotypically unimodal but ecologically variable population of stickleback. Am Nat 172:733-739

Spoon TR, Millam JR, Owings DH (2006) The importance of mate behavioural compatibility in parenting and reproductive success by cockatiels Nymphicus hollandicus. Anim Behav 71:315-326

Sumner MD (2013) R package trip: Spatial analysis of animal track data (version 1.1-17). http://CRAN.R-project.org/ package $=$ trip

Thiebot JB, Bost CA, Dehnhard N, Demongin L and others (2015) Mates but not sexes differ in migratory niche in a monogamous penguin species. Biol Lett 11:20150429 
Tremblay Y, Thiebault A, Mullers R, Pistorius P (2014) Birdborne video-cameras show that seabird movement patterns relate to previously unrevealed proximate environment, not prey. PLOS ONE 9:e88424

Tveraa T, Sæther BE, Aanes R, Erikstad KE (1998) Body mass and parental decisions in the Antarctic petrel Thalassoica antarctica: How long should the parents guard the chick? Behav Ecol Sociobiol 43:73-79

Von Holst D (1998) The concept of stress and its relevance for animal behavior. Adv Stud Behav 27:1-131

Votier SC, Bearhop S, Ratcliffe N, Furness RW (2004) Reproductive consequences for great skuas specializing as seabird predators. Condor 106:275-287

Wagner RH (1999) Sexual size dimorphism and assortative mating in razorbills Alca torda. Auk 116:542-544

Ward P, Zahavi A (1973) The importance of certain assemblages of birds as 'information-centres' for food-finding. Ibis 115:517-534

Editorial responsibility: Francis Daunt (Guest Editor), Penicuik, UK
Watanuki Y (1992) Individual diet difference, parental care and reproductive success in slaty-backed gulls. Condor 94:159-171

* Weimerskirch H, Le Corre M, Ropert-Coudert Y, Kato A, Marsac F (2006) Sex-specific foraging behaviour in a seabird with reversed sexual dimorphism: the red-footed booby. Oecologia 146:681-691

* Weimerskirch H, Bertrand S, Silva J, Marques JC, Goya E (2010) Use of social information in seabirds: compass rafts indicate the heading of food patches. PLOS ONE 5: e9928

* White CR, Butler PJ, Grémillet D, Martin GR (2008) Behavioural strategies of cormorants (Phalacrocoracidae) foraging under challenging light conditions. Ibis 150: 231-239

*Wojczulanis-Jakubas K, Jakubas D, Welcker J, Harding AM and others (2011) Body size variation of a high-Arctic seabird: the dovekie Alle alle. Polar Biol 34:847-854

Submitted: March 30, 2016; Accepted: July 15, 2017

Proofs received from author(s): August 12, 2017 